\author{
Department of Physics and Astronomy \\ Experimental Particle Physics Group \\ Kelvin Building, University of Glasgow, \\ Glasgow, G12 8QQ, Scotland \\ Telephone: +44 (0)1413302000 Fax: +44 (0)1413305881
}

\title{
Design, Simulation, Production and Initial Characterisation of 3D Silicon Detectors
}

\author{
D. Pennicard ${ }^{1}$, G. Pellegrini' ${ }^{2}$, M. Lozano ${ }^{2}$, \\ C. Fleta $^{1}$, R. Bates ${ }^{1}$, C. Parkes $^{1}$ \\ ${ }^{1}$ University of Glasgow, Department of Physics and Astronomy, Glasgow, UK, G12 8QQ \\ ${ }^{2}$ Instituto de Microelectronica de Barcelona, IMB-CNM-CSIC, 08193 Bellaterra, Barcelona, Spain
}

\begin{abstract}
3D detectors are photodiode radiation detectors with n- and p-type electrode columns passing through a silicon substrate. This structure makes it possible to achieve a very small electrode spacing without reducing the sensitive thickness. This greatly reduces the detector's depletion voltage and collection time, and hence improves its radiation hardness. This could make 3D detectors useful as pixel detectors for future high-luminosity colliders, such as the Super-LHC.

The research institute IMB-CNM (Centro Nacional de Microelectronica, Barcelona) have produced 3D pad, pixel and strip detectors with a "double sided 3D" structure. This fabrication has been done alongside design and simulation work at the University of Glasgow. The first devices produced by CNM have been successfully IV and CV tested, and source tests are ongoing. Additionally, this conference record discusses work done by other 3D detector collaborations: Stanford, Manchester University and Sintef; FBK (Trento); and Glasgow, Diamond Light Source and IceMOS Ltd.
\end{abstract}

10th International Conference on Instrumentation for Colliding Beam Physics

Budker Institute of Nuclear Physics, Novosibirsk, Russia 


\section{Introduction}

"3D detectors", proposed by S. Parker et al.[1], are a relatively new variety of silicon photodiode radiation detector. Instead of having $\mathrm{n}$ - and p-type electrodes on their front and back surfaces like a standard photodiode, these sensors have grids of $n$ - and p-type electrode columns passing through the thickness of a silicon substrate. Using this structure makes it possible to reduce the spacing between the electrodes to as little as $30 \mu \mathrm{m}$, while maintaining a reasonable substrate thickness. This leads to a dramatic reduction in the detector's depletion voltage and collection time, without reducing the charge generated in the device by a high-energy particle. Additionally, the device structure reduces charge-sharing between adjacent pixels [2].

The first 3D detectors were produced nearly a decade ago [3], but research into these detectors has increased over the last few years, for a couple of reasons. Firstly, fabricating the electrode columns in these detectors requires the use of specialised micromachining techniques. As these techniques have improved, the idea of producing these detectors on a large scale, with an acceptable cost and reliability, has become more feasible. Secondly, 3D detectors now have a promising application, as radiation-hard detectors in future high-energy physics experiments such as the Super-LHC [4].

\section{3D detectors, radiation hardness and the Super-LHC}

"Super-LHC" is an upgrade for the Large Hadron Collider at CERN, planned for sometime after 2015 [5]. This upgrade would increase the LHC's luminosity by a factor of ten, increasing the number of collisions in order to improve measurements of rare production and decay processes. The innermost layer of the ATLAS pixel detector, just $5 \mathrm{~cm}$ from the beamline, would then receive an equivalent radiation fluence of $1 \times 10^{16}$ $1 \mathrm{MeV}-\mathrm{n}_{e q} / \mathrm{cm}^{2}$ over the SLHC's running time, which is a factor of 10 greater than the current pixel detector is required to withstand [6]. CERN collaborations are researching a variety of radiation-hard detector technologies, including $3 \mathrm{D}$, to meet this challenge.

When high-energy particles pass through a detector, they create defects in the silicon lattice, degrading the material's behaviour [7]. Firstly, some defects act as p-type dopants. At high fluences this causes a large increase in the effective doping concentration (and also type inversion if the substrate is n-type), giving a high depletion voltage. So, standard planar sensors cannot be fully depleted very far beyond $1 \times 10^{15} \mathrm{n}_{e q} / \mathrm{cm}^{2}$. However, because 3D detectors only have to be depleted by the short distance between adjacent columns, they have naturally low depletion voltages, counteracting this effect. Secondly, the defects can also trap the electrons and holes generated by a particle passing through the detector, preventing them from being collected and reducing the readout signal. In a 3D detector these electrons and holes can be collected much more quickly, reducing the charge trapping. The third effect of radiation damage, an increase in leakage current, is normally counteracted by cooling the detector.

\subsection{Simulation of 3D detectors}

To study the behaviour of 3D detectors following radiation damage, simulations were done using "Synopsys TCAD" version Z-2007.03 [8], a finite-element semiconductor simulation package. The effects of radiation damage were taken into account by modelling the defect behaviour directly, as described in [9]. Note that the trapping rates at radiation damage levels beyond $1 \times 10^{15} \mathrm{n}_{e q} / \mathrm{cm}^{2}$ are not well known; this radiation damage model assumes pessimistic values.

Figure 1 compares the simulated charge collection from a p-type double-sided 3D detector (described in section 3.1) using a bias of $100 \mathrm{~V}$ and a pixel size of $55 \mu \mathrm{m}$, with experimental [10] and simulated results from an n-in-p strip detector at $900 \mathrm{~V}$. The simulation of the planar detector gives about $30 \%$ lower charge collection than the real device at high fluences, confirming that the simulated damage models will tend to underestimate CCE. In spite of this, the simulated 3D detector has much higher charge collection than the planar sensor at high damage levels.

In 3D detectors - particularly those with large pixel sizes - a variety of electrode layouts can be used, and the optimum design needs to be chosen. For example, using closely-spaced columns will give lower depletion voltages and less charge trapping, but this will also increase the device's capacitance, increasing the noise level. Additionally, the electrode columns themselves have reduced sensitivity to particles, so the total column volume should be kept small. In [9], this issue is considered more fully. 


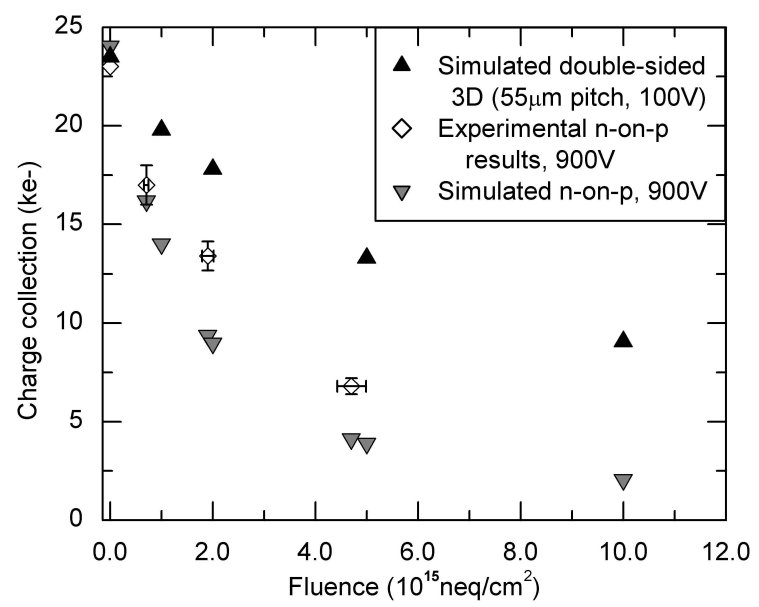

Figure 1: Simulated charge collection from a double-sided 3D detector at 100V, compared to experimental [10] and simulated results from n-on-p planar sensors.

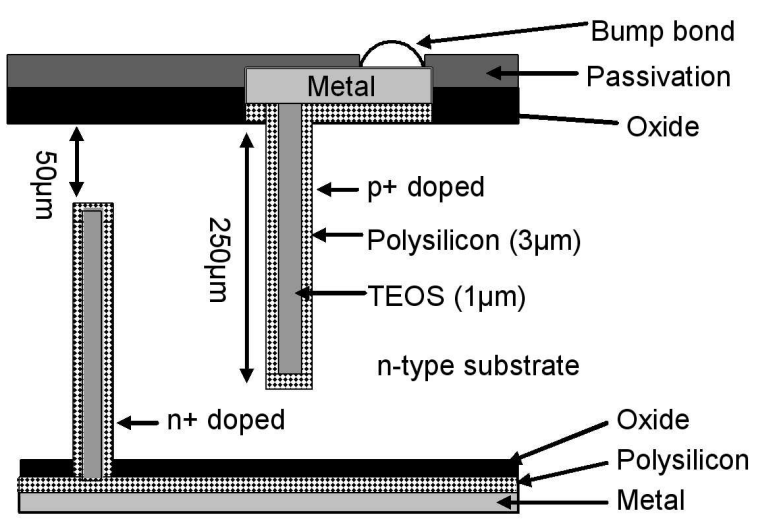

Figure 2: Structure of a double-sided 3D detector.

\section{Double-sided 3D detectors at CNM}

\subsection{Device structure and fabrication}

CNM (Barcelona, Spain) have proposed and fabricated an alternative 3D structure, called double-sided 3D. Instead of fabricating both the $\mathrm{n}+$ and $\mathrm{p}+$ columns from the front side of the detector, one set is fabricated from the front side and used for readout, and the second set is fabricated from the back side and used for biasing, as shown in Fig. 2. The bias columns are connected together by layers of polysilicon and metal on the backside, and the readout columns on the front surface can be connected to the readout chip. In contrast, a standard 3D detector requires routing lines to both bias and read out the sensor from its front surface.

The first production run of these detectors has been completed. The finished wafers contain a variety of devices, including pad and strip detectors, Medipix2 pixels, and test structures. The devices were fabricated on a $300 \mu \mathrm{m}$-thick n-type substrate. The electrode columns are $10 \mu \mathrm{m}$ in diameter, and $250 \mu \mathrm{m}$ long, so they do not pass through the full substrate thickness. Simulations [11] show that these devices will behave very similarly to full 3D detectors, except that the electric field and collection time will be poorer around the front and back surfaces of the device. In future production runs, both n- and p-type substrates will be used, ATLAS pixels will be included in the wafers, and the columns will pass through the full substrate thickness.

Like in a standard 3D detector, the electrode columns were produced by first etching holes in the substrate, then filling them [3]. The etching was done with an Inductively-Coupled Plasma (ICP) etching process, using an aluminium/copper mask to protect the unetched areas. Unlike chemical etching, this process gives a very directional etch, producing deep holes in the substrate. The maximum possible depth of a hole is dependent on its diameter - with $10 \mu \mathrm{m}$ diameter, a depth of $250 \mu \mathrm{m}$ can currently be achieved. After the etching, $3 \mu \mathrm{m}$ of polysilicon was deposited inside the holes, and then the holes were doped through the polysilicon with boron 


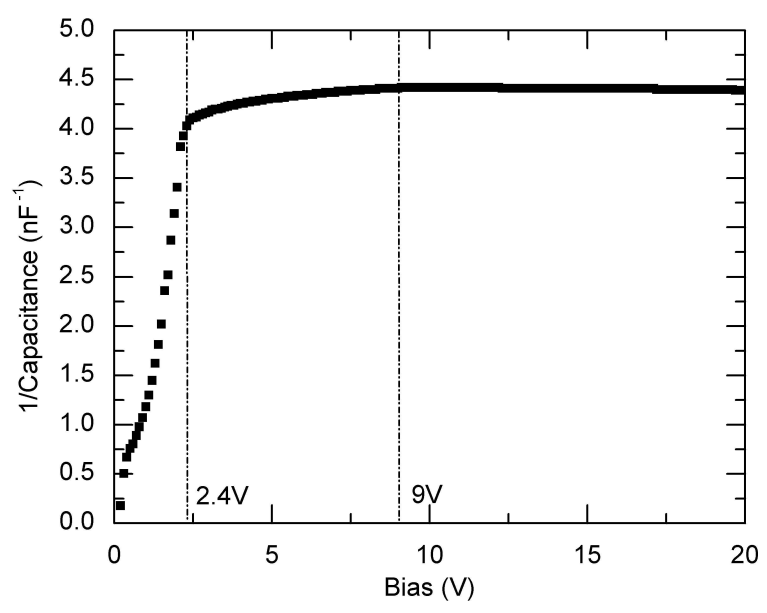

Figure 3: Inverse capacitance characteristics of a 3D pad detector with 90 by 90 columns and $55 \mu \mathrm{m}$ pitch.

or phosphorus. This gave a doping profile with a depth of about $2 \mu \mathrm{m}$. The interior was then passivated with a layer of silica, deposited using TEOS. These steps were repeated twice to produce the n-type and p-type holes. The double-sided process has the advantage that these steps are only carried out once on each side, starting from a virtually untouched surface each time, rather than twice on the front surface. However, double-sided alignment is needed.

\subsection{Preliminary tests of double-sided 3D detectors}

The finished devices have undergone IV and CV testing using a Cascade Microtech probe station. These tests have confirmed that the diode junctions in the devices work correctly.

Due to the devices' unusual structure, their depletion behaviour differs from a normal photodiode. Capacitancevoltage measurements were made at $10 \mathrm{kHz}$ on a pad detector with a 90 by 90 grid of $\mathrm{p}+$ and $\mathrm{n}+$ columns. The pitch between columns of the same type is $55 \mu \mathrm{m}$, giving a total area of $0.245 \mathrm{~cm}^{2}$. Fig. 3 shows the inverse of the capacitance versus bias; this was chosen to make small changes in capacitance more visible. In simulations [11], the depletion region grows outwards from each $\mathrm{p}+$ column as the bias increases, reaching the adjacent $\mathrm{n}+$ columns at just $2 \mathrm{~V}$. At this point, the full device is depleted, apart from around the back surface. Because the $\mathrm{p}+$ columns do not reach the back surface, a higher bias of around $8 \mathrm{~V}$ is required to deplete this region. Looking at Fig. 3, the capacitance changes dramatically over the first $2.4 \mathrm{~V}$, as most of the device volume depletes, but only reaches a minimum (i.e. $1 / \mathrm{C}$ reaches a maximum) around $9 \mathrm{~V}$ as the back surface depletes.

Next, sets of strip detectors were IV-tested. These detectors had 50 strips, each containing $50 \mathrm{p}+$ columns. The column pitch was $80 \mu \mathrm{m}$, giving a strip length of $4 \mathrm{~mm}$. During testing, probe needles at $0 \mathrm{~V}$ were placed on three adjacent strips and on the guard ring, and the device was biased from the back side. The resulting currents for a typical strip test are shown in Fig. 4. Four strip detectors were tested, and all the strips had currents of around $100 \mathrm{pA}$ at $21^{\circ} \mathrm{C}$. All the strips tested could be reliably biased up to $50 \mathrm{~V}$ without breakdown, which is large compared to the $2.4 \mathrm{~V}$ required to deplete between the columns. However, a large difference was seen in the guard ring current in the 4 detectors, which ranged from $0.03 \mu \mathrm{A}$ (measured at $50 \mathrm{~V}$ ) to a maximum of $20 \mu \mathrm{A}$ (measured at 10V). Since the guard ring current is mainly caused by surface leakage, differences in the cut edges of the samples may be responsible. The high guard current does not prevent the strips from operating, but it does increase power dissipation. When $\mathrm{C}-\mathrm{V}$ tests were done on these sensors, the capacitance was around $5 \mathrm{pF}$ per $4 \mathrm{~mm}$ strip. This is comparatively large (ATLAS SCT strips are designed to have less than $2.2 \mathrm{pF} / \mathrm{cm}$ capacitance) because the $\mathrm{p}+$ and $\mathrm{n}+$ electrodes are closer together in a $3 \mathrm{D}$ device than in a standard planar detector.

Currently, the strip detectors are having their charge collection efficiency tested using a beta source and $\mathrm{LHCb}$ readout electronics. The IV, CV and CCE tests will also be repeated after the devices have been irradiated, to evaluate their radiation hardness. Additionally, a set of 3D Medipix2 devices will be tested in an $\mathrm{X}$-ray beamline, and testbeams at CERN are planned for the summer. 


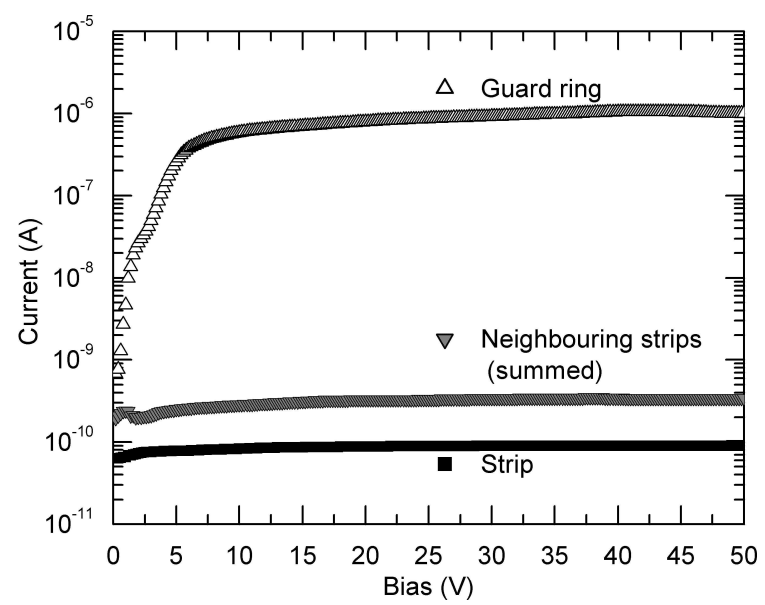

Figure 4: Current-voltage characteristics of a 3D strip detector with $80 \mu \mathrm{m}$ pitch and $4 \mathrm{~mm}$ strip length, tested at $21^{\circ} \mathrm{C}$.

\section{Other 3D detector projects}

\subsection{Stanford, Manchester and Sintef}

The first 3D detectors were produced at the Stanford Nanofabrication Facility [3], and currently the University of Manchester and CERN are collaborating to test 3D detectors fabricated at Stanford. These tests have included charge collection measurements on heavily irradiated ATLAS 3D detectors [12]. Even at a damage fluence of $8.81 \times 10^{15} \mathrm{n}_{e q} / \mathrm{cm}^{2}$, close to the maximum fluence expected at SLHC, these sensors are still able to obtain a good charge collection of 13200 electrons and a signal-to-noise ratio of 46:1.

The Stanford 3D devices use two sets of electrode columns passing through the full substrate thickness, fabricated from the front surface. Additionally, the Stanford devices use an "active edge" electrode [13]. Instead of simply dicing the finished detector wafer with a saw, the wafer is mounted on a support wafer during fabrication, the edges of the sensors are plasma etched, and then a layer of doped polysilicon is added to the edges. (This uses the same tools required for hole etching and filling.) The doped polysilicon edge can function as an electrode, reducing the dead area at the edge of the detector to just $5 \mu \mathrm{m}$, compared with $100 \mu \mathrm{m}$ or so in a typical detector. This would make it possible to build a full pixel detector system using fewer overlapping sensors.

Currently, Stanford and Manchester are also working with Sintef, an independent research organisation in Norway, to reproduce the Stanford fabrication process in Sintef's cleanrooms. This should allow larger-scale production of these sensors.

\subsection{FBK (Trento)}

Fondazione Bruno Kessler (FBK, former ITC-irst) is a research institute in Trento, Italy. In collaboration with INFN, they have produced double-sided double-column 3D detectors, with n-type substrates and $\mathrm{p}+$ column readout. These devices have a similar structure to CNM's double-sided 3D detectors, except that in addition to the $\mathrm{n}+$ bias columns, the entire back surface of the detector has n-type doping. The detectors tested so far have shown good C-V and I-V characteristics [14]. More devices with p-substrates and $\mathrm{n}+$ readout are currently being produced. Previously, FBK have also produced and tested simpler "single-type column" 3D devices in collaboration with CNM, with $\mathrm{n}+$ columns etched from the front surface of the detector, and a blanket $\mathrm{p}+$ implant on the back surface [15].

\subsection{Glasgow, Diamond Light Source, and IceMOS Ltd}

In addition to their applications in high-energy physics, 3D detectors are potentially useful for X-ray diffraction experiments in new synchrotrons such as Diamond Light Source (UK). When coupled to a single-photon counting readout chip, such as Medipix2 [16], photodiode detectors can give a high signal-to-noise ratio, a short readout time, and a large dynamic range. 3D detectors have the additional benefits of low charge sharing, the possibility of using thick substrates while maintaining good performance (though this depends on how deep the columns can be etched), and also a reduced dead area when active edges are used [2]. 
Currently, Glasgow are working with Diamond Light Source to develop 3D sensors for X-ray detection. The fabrication is being done at IceMOS Ltd, a semiconductor fabrication company in Belfast. These would be the first 3D detectors produced entirely in industry. The sensors are being designed and tested at the University of Glasgow. Currently, IceMOS have finished a prototype run of detectors. Some of these sensors have been IV and CV tested, but there are reliability problems. A second production run is being started, with an improved fabrication flow.

\section{Conclusions}

As part of the drive to develop radiation-hard pixel detectors for the Super-LHC, a few institutions are currently producing 3D detectors. CNM have completed their first set of these detectors, which show good I-V and C-V characteristics. Further experimental tests and a new production run at CNM are ongoing.

\section{Acknowledgements}

This work has been supported by the Spanish Ministry of Education and Science through the GICSERV program "Access to ICTS integrated nano- and microelectronics cleanroom". This work was carried out in the context of the RD50 collaboration.

\section{References}

[1] S. I. Parker, C. J. Kenney, J. Segal, Nucl. Instr. and Meth A 395 (3) (1997) 328-343.

[2] V. A. Wright et al., IEEE Trans. Nucl. Sci. 52 (5) (2005) 1873-1876.

[3] C. Kenney, S. Parker, J. Segal, C. Storment, IEEE Trans. Nucl. Sci. 46 (4) (1999) 1224-1236.

[4] C. Da Via et al., Nucl. Instr. and Meth A 509 (1-3) (2003) 86-91.

[5] F. Gianotti, Nuclear Physics B-Proceedings Supplements 147 (2005) 23-32.

[6] Y. Unno, Nucl. Instr. and Meth A 569 (1) (2006) 41-47.

[7] V. Eremin, E. Verbitskaya, Z. Li, Nucl. Instr. and Meth A 476 (3) (2002) 537-549.

[8] Synopsys Inc., Synopsys TCAD manuals, http://www.synopsys.com/products/tcad/tcad.html, (2007).

[9] D. Pennicard et al., Nucl. Instr. and Meth A, In Publication, doi:10.1016/j.nima.2008.03.100

[10] P. P. Allport, G. Casse, M. Lozano, P. Sutcliffe, J. J. Velthuis, J. Vossebeld, IEEE Trans. Nucl. Sci. 52 (5) (2005) 1903-1906.

[11] D. Pennicard et al., IEEE Trans. Nucl. Sci. 54 (4) (2007) 1435-1443.

[12] C. Da Via et al., Nucl. Instr. and Meth A 587 (2-3) (2008) 243-249.

[13] C. Da Via, J. Hasi, C. Kenney, A. Kok, S. Parker, Nucl. Instr. and Meth A 549 (1-3) (2005) 122-125.

[14] G.-F. Dalla Betta, et al., 2007 IEEE Nuclear Science Symposium Conference Record (2007) 983-987

[15] C. Piemonte, M. Boscardin, G. F. D. Betta, S. Ronchin, N. Zorzi, Nucl. Instr. and Meth A 541 (1-2) (2005) 441-448.

[16] X. Llopart, M. Campbell, R. Dinapoli, D. S. Segundo, E. Pernigotti, IEEE Trans. Nucl. Sci. 49 (5) (2002) $2279-2283$. 